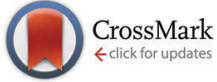

Cite this: Phys. Chem. Chem. Phys., 2015, 17, 22846

Received 13th May 2015, Accepted 6th August 2015

DOI: $10.1039 / c 5 c p 02767 e$

www.rsc.org/pccp

\section{Non-innocent adsorption of Co-pyrphyrin on rutile(110) $\dagger$}

\author{
Yeliz Gurdal,* Sandra Luber, Jürg Hutter and Marcella Iannuzzi
}

Solar-light driven water splitting is a promising way for the sustainable production of molecular hydrogen, the latter representing an efficient carrier for energy storage and conversion into common liquid fuels. In search of novel catalysts for high-performance water splitting devices, Co-pyrphyrin (CoPy) has been recently synthesized and successfully used as a homogeneous water reduction catalyst. We investigate the adsorption of this molecule on the rutile $\mathrm{TiO}_{2}(110)$ surface as a possible first step towards the design of a heterogeneous water reduction system. We find that the adsorption of the molecule is stabilized by the interaction of the cyano groups with the under-coordinated Ti centers present at the surface. This interaction induces the rehybridization of the molecular orbitals localized on the cyano groups and the realignment of the lowest unoccupied molecular states. Moreover, the highest occupied molecular orbital of CoPyarutile(110) is localized on CoPy and the energy gap turns out to be significantly smaller than the gap of pristine rutile(110). This implies that direct or indirect injection of electrons from CoPy to the rutile(110) surface is in principle possible upon the absorption of light in the visible range. On the other hand, the electronic properties of the Co(॥) center are not modified by the adsorption, which suggests that CoPy and its derivatives may be used in water electrolysis for hydrogen production also in the adsorbed state.

\section{Introduction}

In the quest for renewable energy sources molecular hydrogen is one of the most favoured candidates as an energy carrier. Compared to other means, such as electricity, bio-fuels, or conventional fuels, hydrogen has the highest specific energy content, ${ }^{1}$ and is also environmentally preferable, because the waste product of its reaction with pure oxygen is simply water. ${ }^{2}$ However, the large scale production and storage of molecular hydrogen is still an open issue. Several techniques can be employed for its production, such as water electrolysis, ${ }^{3}$ steam and ethanol reforming, ${ }^{4,5}$ partial oxidation of hydrocarbons, ${ }^{6}$ dark fermentation, ${ }^{7}$ and photo-chemical water reduction. The latter is of particular interest, because it emulates natural processes and needs only solar light as an energy source. ${ }^{8}$

Water reduction in a homogeneous environment has been widely studied. ${ }^{9}$ Most of the working systems are based on the use of expensive metals as catalytic reduction centers. ${ }^{10} \mathrm{How}^{-}$ ever, it has been shown that also less expensive Co-based catalysts can be employed for $\mathrm{H}_{2}$ production with low overpotentials. ${ }^{11}$ Peters et al. ${ }^{12}$ investigated the catalytic activity of

Institut für Chemie, Universität Zürich, Winterthurerstrasse 190, CH-8057 Zürich, Switzerland.E-mail: yeliz.guerdal@chem.uzh.ch, sandra.luber@chem.uzh.ch, hutter@chem.uzh.ch, marcella.iannuzzi@chem.uzh.ch

$\dagger$ Electronic supplementary information (ESI) available. See DOI: 10.1039/c5cp02767e several cobalt complexes with $\mathrm{BF}_{2}$ glyoxime or propane bridged tetraimine ligands in acetonitrile. They concluded that with less electron donating ligands the reduction of Co-complexes can be achieved easily, leading to $\mathrm{H}_{2}$ production. Guttentag et $a{ }^{13}$ studied the activity of $\left[\mathrm{Co}^{\mathrm{III}} \mathrm{Br}_{2}(\mathrm{DO})(\mathrm{DOH}) \mathrm{pn}\right]$ with a photo-sensitizer $\left[\operatorname{Re}(\mathrm{CO})_{3}(\text { bipy })(\mathrm{py})\right]^{+}$in a pure water and an acidic environment, demonstrating that $\mathrm{H}_{2}$ production is possible without organic solvents. Macro-cyclic molecules like metal centered porphyrins are also widely studied alternative catalysts, thanks to their low cost and wide range of light absorption capability. ${ }^{14,15}$ Solis et al. ${ }^{16}$ modeled cobalt hangman porphyrins in acetonitrile solution and determined the pathway for proton coupled electron transfer for $\mathrm{H}_{2}$ generation. For recent reviews and studies on homogeneous water reduction we refer to ref. 17-20.

In spite of the established achievements in $\mathrm{H}_{2}$ generation in homogeneous environments, the most important drawback is the rapid recombination of photo-generated products in solution. $^{21}$ Therefore, it has been proposed that in heterogeneous environments, where the catalyst is combined with a supporting material, the fast back electron transfer can be prevented and the system is stabilized. ${ }^{22}$ Several studies are already available on the properties of metal centered porphyrins adsorbed on $\mathrm{TiO}_{2}$ surfaces. ${ }^{23-25}$

Only relatively few studies on the combination of Co-complexes with heterogeneous systems have been published. The Co-based 
complex $\left[\mathrm{Co}^{\mathrm{III}}(\mathrm{dmgH})_{2}\right.$ (pyridyl-4-hydrophosphonate)Cl] adsorbed on a $\mathrm{TiO}_{2}$ surface via anchoring groups has been considered as a catalyst for proton reduction. ${ }^{26}$ Yin $e t ~ a l .{ }^{27}$ studied the organic dye eosin Y (EY) and several other Co complexes also adsorbed on the $\mathrm{TiO}_{2}$ surface through different anchoring groups. They concluded that $\mathrm{TiO}_{2}$ is able to harvest electrons from the excited EY molecules, thus generating long-lived charge-separated states, which eventually lead to the electron transfer to the Co-catalyst and the reduction process. A similar reductive pathway has also been observed by Lakadamyali et al., ${ }^{28}$ who studied the co-adsorption of the CoP catalyst and a ruthenium dye RuP on $\mathrm{TiO}_{2}$. The photo-excitation of RuP induces an electron injection into the conduction band of $\mathrm{TiO}_{2}$. The photoelectrons are then transferred to $\mathrm{CoP}$, where the $\mathrm{H}_{2}$ formation occurs. The photo-excitation of $\mathrm{Co}(\mathrm{I})$-porphyrin on $\mathrm{TiO}_{2}$ in acetonitrile-pyridine solution has also been studied ${ }^{29}$ showing that after the rapid electron injection from $\mathrm{Co}$ to $\mathrm{TiO}_{2}$ and the coordination of pyridine to $\mathrm{Co}$, the undesired charge recombination is significantly slowed down.

Among the heterocyclic molecules, the porphyrin derived tetra-aza macro-cycle, constituted of four pyridine rings, is named pyrphyrin (Py) but also known as an Ogawa porphyrin like ligand. Pyrphyrin has been introduced first by Ogawa et $a .^{30}$ in 1984. Later, alkylated pyrphyrin has also been synthesized and its optical properties have been characterized. ${ }^{31}$ More recently, metal-centered pyrphyrins have been studied both experimentally and theoretically to determine their UV-visible spectra in solution. ${ }^{32-34}$

The need for new, promising, and feasible photo-catalysts for water reduction and the unexplored potential of pyrphyrin adsorbed on the $\mathrm{TiO}_{2}$ surface are the motivations for the present work. To the best of our knowledge, this is the first time that pyrphyrin on semiconductors is investigated. It is generally accepted that anatase phase of $\mathrm{TiO}_{2}$ exhibits higher photocatalytic activity than rutile $\mathrm{TiO}_{2},{ }^{35}$ which resulted in theoretical investigation of water- $\mathrm{TiO}_{2}$ interactions mostly with the anatase phase. ${ }^{36}$ However, in this study only the rutile phase is considered, which is easier to obtain as a high quality single crystal ${ }^{37}$ and, for this reason, it is going to be employed in upcoming experimental studies of the catalytic activity of supported-CoPy.

We study the structural and electronic properties of the complex, CoPy, adsorbed on the rutile(110) surface, by means of density functional theory (DFT). In the following, we first briefly describe the applied methodology and the models used for the molecule, the surface and the complex. Next, we discuss the possible adsorption configurations of CoPy@rutile(110), and finally we analyze in detail the electronic structure of the most stable configuration.

\section{Computational methodology}

Electronic structure calculations are performed at the KohnSham DFT level, employing the Gaussian and plane wave (GPW) formalism as implemented in the CP2K/QUICKSTEP package. ${ }^{38}$ Double-zeta valence plus polarization (DZVP) basis sets, optimized on molecular geometries (Mol-Opt method), ${ }^{39}$ are employed for all atomic kinds. A cutoff of $600 \mathrm{Ry}$ is used for the auxiliary plane wave basis set. The interaction between the valence electrons and the atomic cores is described through norm-conserving Goedecker-Teter-Hutter (GTH) pseudo potentials. ${ }^{40}$ The valence shells contain $12,6,17,4,1$, and 5 electrons for Ti, O, Co, C, H, and N, respectively. Periodic boundary conditions are always applied.

As exchange-correlation functional, the general gradient approximation (GGA) by Perdew-Burke-Ernzerhof (PBE) ${ }^{41}$ is used. The dispersion interactions are corrected according to the Grimme-D3 scheme ${ }^{42}$ for the optimization of the geometries. This level of theory has been proved to be sufficiently accurate to describe the structural properties of the materials involved in this study. Namely, the lattice parameters of bulk rutile turn out to be in agreement with the experimental values within an error of $1 \%{ }^{43}$ However, PBE is known to underestimate the energy gap of $\mathrm{TiO}_{2}$ as for other oxides. ${ }^{44,45}$ The most commonly adopted solution to this deficiency is the use of suitable hybrid functionals that can reproduce the experimental value. ${ }^{46,47}$ For the specific case of $\mathrm{TiO}_{2}$, we find, in agreement with previous studies, that the best accuracy in the energy gap is achieved by applying the Heyd-ScuseriaErnzerhof (HSE06) hybrid functional. ${ }^{43,48,49}$ All hybrid functional calculations are performed with the help of the auxiliary density matrix method (ADMM) ${ }^{50-52}$ which provides a significant speed up and makes it possible to compute the electronic structure of several hundreds of atoms of condensed matter systems. The required ADMM basis sets have been optimized to reproduce the results of full hybrid functional calculations with very high accuracy. Despite the speed up obtained with ADMM, hybrid functional geometry optimization is still computationally very demanding and does not produce important changes with respect to the PBE optimization. Therefore, our strategy consists in carrying out the refinement of the electronic structure of PBE-optimized geometry using the HSE06 hybrid functional.

\section{Structural models}

\section{A. Rutile: bulk and (110) surface}

The rutile lattice belongs to the $P 4_{2} / m n m$ (No. 136) tetragonal space group. ${ }^{53,54}$ In the bulk, each Ti atom is coordinated by six $\mathrm{O}$ atoms, while the $\mathrm{O}$ atoms are three-fold coordinated by Ti. In order to assess our PBE computational set up, the lattice parameters are optimized for a $3 \times 3 \times 3$ supercell (162 atoms). This supercell is sufficiently large to converge the bulk structural properties, which turn out to be in very good agreement with previously published data, from both experimental and DFT studies (see Table 1). The corresponding band gap energies, $E_{\text {gap }}$, computed for the PBE and the HSE06 functionals are reported in Table 2 and also compared to previous studies. All GGA and LDA results underestimate $E_{\text {gap }}$ by more than $1.2 \mathrm{eV}$, while PBE0 overestimates it by about the same amount. Only 
Table 1 Lattice parameters ( $a$ and $c$ ) and bulk modulus $(B)$ of bulk $\mathrm{TiO}_{2}$ The values reported for this work are obtained for the $3 \times 3 \times 3$ supercell and the PBE functional

\begin{tabular}{lllll}
\hline Method & $a[\AA]$ & $c[\AA]$ & $B[\mathrm{GPa}]$ & Ref. \\
\hline GGA/PBE/GPW & 4.606 & 2.966 & 219 & This work \\
GGA/PW92/PAW & 4.649 & 2.972 & 225 & 55 \\
GGA/PBEsol/PW & 4.604 & 2.947 & - & 56 \\
LDA/PAW & 4.554 & 2.927 & 256 & 57 \\
Experiment & 4.587 & 2.954 & 210 & 58 \\
Experiment & 4.594 & 2.959 & - & 53
\end{tabular}

Table 2 Band gap energy $\left(E_{\text {gap }}\right)$ of bulk $\mathrm{TiO}_{2}$. The values reported for this work are obtained for the $3 \times 3 \times 3$ supercell

\begin{tabular}{lll}
\hline Method & $E_{\text {gap }}[\mathrm{eV}]$ & Ref. \\
\hline GGA/PBE & 1.73 & This work \\
GGA/PBE & 1.74 & 59 \\
GGA/PW92 & 1.80 & 57 \\
LDA & 1.81 & 57 \\
HSE06 & 3.35 & This work \\
HSE06 & 3.11 & 43 \\
PBE0 & 4.21 & 43 \\
Experiment & 3.0 & 58
\end{tabular}

the value obtained with HSE06 approximates the experiment with reasonable accuracy. The slight discrepancies between results obtained with the same functional can be ascribed to different basis sets and pseudopotentials.

Fig. 1 shows projected density of states (PDOS) for $\mathrm{O}$ and $\mathrm{Ti}$ atoms in bulk rutile. The highest occupied states have O-2p

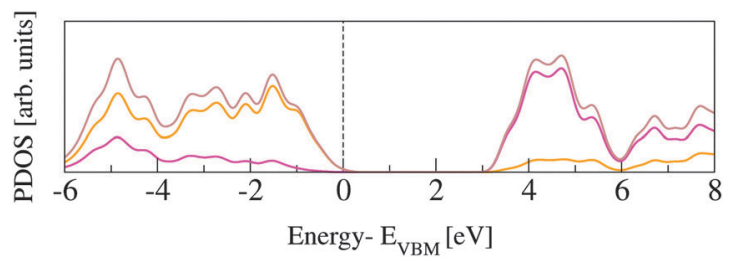

Fig. 1 Density of states of bulk rutile: total DOS plotted with brown line, O PDOS in orange, Ti PDOS in magenta. The distributions are normalized by the number of atoms and the aligned at zero with the valence band maximum (VBM) energy. character, whereas the lowest unoccupied states are Ti-3d, in agreement with previously published calculations. ${ }^{60,61}$ The hybridization between $\mathrm{O}-2 \mathrm{p}$ and $\mathrm{Ti}-3 \mathrm{~d}$ states results in covalent bonding. ${ }^{62}$

The (110) surface is the most stable surface of rutile, and also the most frequently studied and used one for applications. ${ }^{63}$ To model such a surface, a symmetric slab consisting of a few atomic layers is cut out from the bulk cleaving along the (110) plane. Above the surface, a sufficiently large amount of vacuum space $(20 \AA)$ is added to avoid interactions with the periodic images in the perpendicular direction. The surface unit cell contains two Ti atoms, which lay on the same plane, and four $\mathrm{O}$ atoms, two on the same plane as $\mathrm{Ti}$, one above (sticking out O) and one below. One surface Ti atom is still sixfold coordinated, as in the bulk, while the second is five-fold coordinated. Among the $\mathrm{O}$ atoms, three are still three-fold coordinated, while the sticking out one is two-fold coordinated. This latter dangling $\mathrm{O}$ atom is a particularly active species, which can lead to the formation of vacancies at the surface, or be saturated. Fig. 2 displays the top and side view of a $3 \times$ 7 slab, containing five atomic layers. We label the surface threefold and two-fold coordinated $\mathrm{O}$ atoms with $\mathrm{O}_{3 \mathrm{c}}$ and $\mathrm{O}_{2 \mathrm{c}}$, while the bulk oxygens are labeled $\mathrm{O}_{3 \mathrm{c} \text {-bulk }}$. We also distinguish between the bulk $\mathrm{Ti}$ atoms, labeled $\mathrm{Ti}_{6 \mathrm{c} \text {-bulk }}$, and the undercoordinated $\mathrm{Ti}$ atoms at the surface, $\mathrm{Ti}_{5 \mathrm{c}}$. In order to determine the optimal number of layers for the slab model, we tested the convergence of the surface energy. The surface energy is calculated as

$$
E_{\text {surface }}=\frac{E_{\text {slab }}-N \cdot E_{\text {bulk }}}{2 \cdot A}
$$

where $E_{\text {slab }}$ is the total energy of the optimized slab model, $E_{\text {bulk }}$ is the energy per $\mathrm{TiO}_{2}$ unit in the bulk, $N$ is the number of units contained in the slab, and $A$ is the area of the exposed surface.

Slabs from 3 to 9 layers have been considered (see ESI $\dagger$ ). We conclude that a 5 layer slab is sufficient to model the rutile(110) surface. The surface energy of the $3 \times 3 \times 5$ slab is $0.72 \mathrm{~J} \mathrm{~m}^{-2}$, in quite good agreement with the values found in the literature. ${ }^{63}$

The band gap calculated by HSE06 for the rutile slab of five layers, where both exposed surfaces are fully relaxed, is $3.18 \mathrm{eV}$, in agreement with the experiment. ${ }^{64}$ The reduction of the
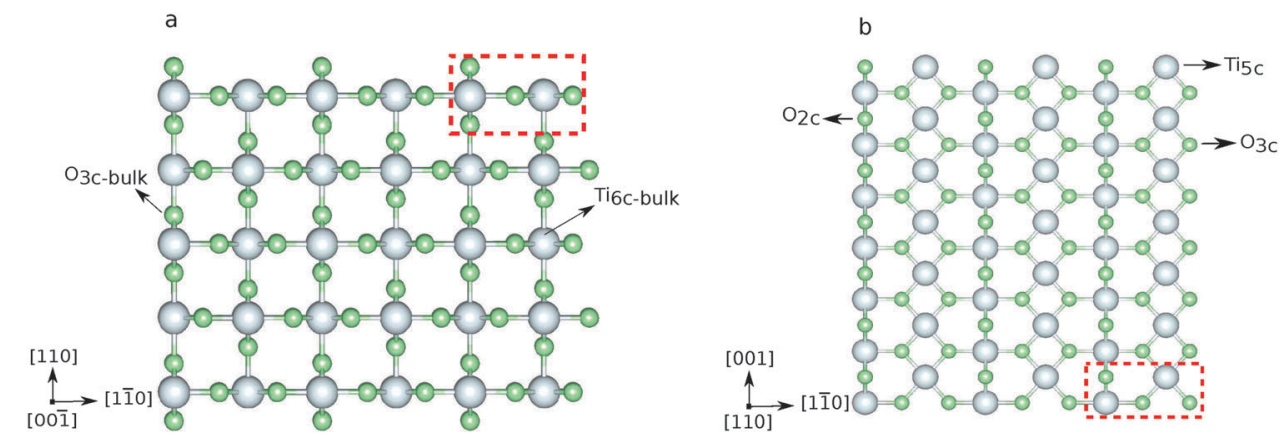

Fig. 2 Ball and stick representation of the rutile(110) $3 \times 7 \times 5$ slab, where 5 is the number of layers: (a) side view and (b) top view. For clarity, only the

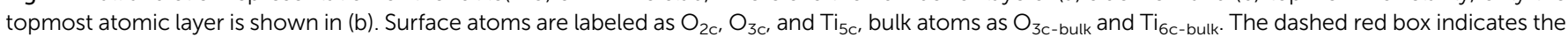
surface unit cell. Color code: light gray: Ti, light green: $\mathrm{O}$. 
energy gap at the surface is due to the presence of energy states in the gap, which are generated by electrostatic shifting of $\mathrm{Ti}_{5 \mathrm{c}}$ states towards $\mathrm{O}_{2 \mathrm{c}}$ and $\mathrm{O}_{3 \mathrm{c} \text {-bulk }}$ bands, also discussed in ref. 65 . We also computed the electronic properties for slabs of different sizes, concluding that the slab of five layers is a good compromise between accuracy and efficiency, see ESI. $\dagger$

\section{B. Pyrphyrin}

Ball and stick sketches of Py and CoPy are shown in Fig. 3. Py is characterized by $\mathrm{CN}$ groups binding to the pyridine rings via $\mathrm{sp}^{2}$ hybridized bridging carbons. We label the nitrogen atoms of the cyano groups $\mathrm{N}_{\mathrm{CN}}$ to distinguish them from the pyridine nitrogens at the center of the molecule $\left(\mathrm{N}_{\mathrm{p}}\right)$. Two $\mathrm{N}_{\mathrm{p}}$ are protonated in Py at anti positions, ${ }^{66}$ while the protons are replaced by the $\mathrm{Co}(\mathrm{II})$ cation in CoPy. In the gas phase, both molecules are planar with a vanishing dipole, as discussed in ref. 34. In the center of the molecule, the four $\mathrm{N}_{\mathrm{p}}$ are symmetrically coordinating Co in CoPy, while the protonated nitrogens in Py are displaced outwards. Along the axis of the cyano groups, $d_{\mathrm{CN}}$, the molecule is slightly shorter for CoPy with respect to Py, due to the interaction between $\mathrm{Co(II)}$ and the electronegative nitrogens. Some structural parameters are reported in Table 3.

Table 4 shows the energy gaps between the highest occupied molecular orbital (HOMO) and the lowest unoccupied molecular orbital (LUMO) as computed for Py and CoPy with the PBE and HSE06 functionals. The presence of Co(II) slightly reduces the energy gap; the effect is more pronounced with HSE06, for more discussion see ESI. $\dagger$ Calculations with CoPy are always
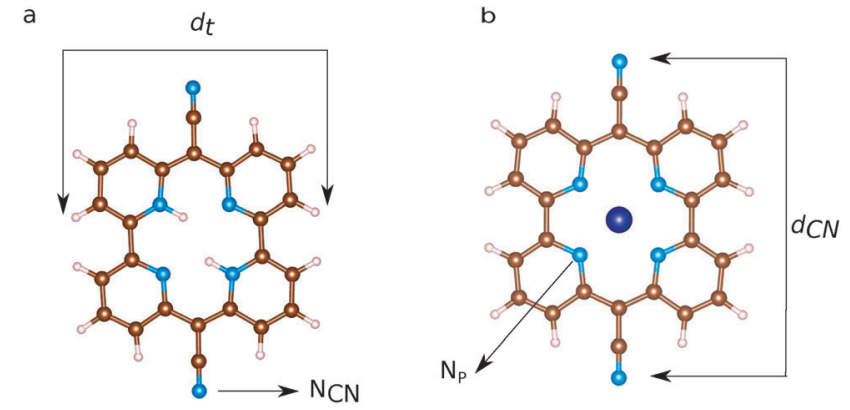

Fig. 3 Ball and stick representations of (a) Py and (b) CoPy. The molecular size is described by means of the two diameters, $d_{\mathrm{CN}}$ and $d_{\mathrm{t}}$. Nitrogens in cyano groups and pyridine rings are labeled as $\mathrm{N}_{\mathrm{CN}}$ and $\mathrm{N}_{\mathrm{p}}$, respectively. Color code: brown: $\mathrm{C}$, light pink: $\mathrm{H}$, dark blue: Co, blue: $\mathrm{N}$.

Table 3 Selected distances calculated for the optimized geometries of Py and CoPy in the gas phase

\begin{tabular}{llc}
\hline Molecule & Distances & Length [̊] \\
\hline Py & $d_{\mathrm{CN}}$ & 11.96 \\
& $\mathrm{~N}_{\mathrm{p}}-\mathrm{N}_{\mathrm{p}}$ (prot.) & 3.98 \\
& $\mathrm{~N}_{\mathrm{p}}-\mathrm{N}_{\mathrm{p}}$ (not prot.) & 3.80 \\
& $d_{\mathrm{t}}$ & 9.45 \\
& & \\
CoPy & $d_{\mathrm{CN}}$ & 11.78 \\
& $\mathrm{~N}_{\mathrm{p}}-\mathrm{N}_{\mathrm{p}}$ & 3.77 \\
& $d_{\mathrm{t}}$ & 9.57
\end{tabular}

Table 4 HOMO-LUMO gap $\left(E_{\text {gap }}\right)$ of Py and CoPy calculated with different density functionals

\begin{tabular}{lll}
\hline Molecule & Functional & $E_{\text {gap }}[\mathrm{eV}]$ \\
\hline Py & PBE & 1.52 \\
Py & HSE06 & 2.23 \\
CoPy & PBE & 1.46 \\
CoPy & HSE06 & 2.03
\end{tabular}
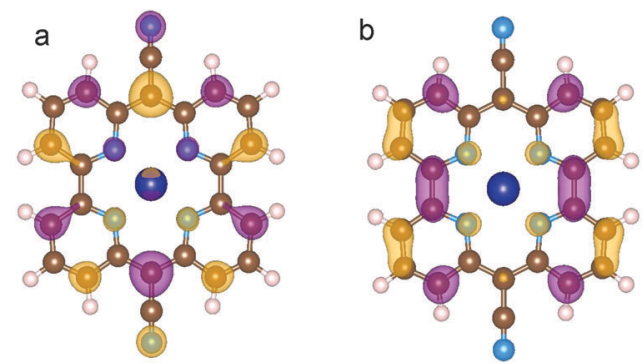

Fig. 4 HOMO (a) and LUMO (b) of CoPy in the gas phase. The displayed isosurfaces are obtained at the value of \pm 0.236 e $\AA^{-3}$.

performed with spin polarization. The lowest energy spin state turns out to be a doublet and the spin density is localized exclusively at the Co center. The HOMO of CoPy in the gas phase has major contributions from the cyano groups, the pyridine carbons, and the $\mathrm{N}_{\mathrm{p}}$, but it is also present at $\mathrm{Co}$ (II) centers. The LUMO, instead, is uniformly distributed over the pyridine $\mathrm{CH}$ groups, the central $\mathrm{C}$ atoms, and the $\mathrm{N}_{\mathrm{p}}$, but it does not have significant contributions from the cyano groups and the $\mathrm{Co}(\mathrm{II})$. The two frontier orbitals of the gas phase molecule are displayed in Fig. 4.

\section{CoPy adsorption on rutile(110)}

In order to find possible adsorption sites of CoPy on rutile, it is necessary to explore the potential energy surface of the CoPy@rutile complex. We started eleven independent optimizations, changing the initial coordinates of the gas-phase optimized CoPy on rutile. The molecule has always been positioned parallel to the surface at a distance a few $\AA$ apart from the topmost atoms moving it rigidly by changing the position of $\mathrm{Co}$ (II) and the two $\mathrm{N}_{\mathrm{CN}}$ with respect to the surface $\mathrm{O}_{2 \mathrm{c}}$ and $\mathrm{Ti}_{5 \mathrm{c}}$ atoms and rotating the molecular axis parallel to the surface (more details in the ESI $\dagger$ ). The optimization is carried out by relaxing the molecular geometry and the two top-most layers of the rutile slab. The other three slab layers are kept fixed at bulk coordinates. From these eleven starting positions, only four distinct stable configurations have been obtained. These four structures are displayed in Fig. 5 and are named model-a, b, c, and $\mathrm{d}$. The adsorption energy $\left(E_{\text {ads }}\right)$ is calculated as

$$
E_{\text {ads }}=E_{\text {complex }}-\left(E_{\text {slab }}+E_{\text {mol }}\right)
$$

where $E_{\text {complex }}$ is the total energy of the complex, $E_{\text {slab }}$ is the energy of the slab where only two layers are fully optimized, and $E_{\text {mol }}$ is the energy of the molecule optimized in a vacuum. Negative values of $E_{\text {ads }}$ correspond to exothermic adsorption. For each optimized structure, the amount of dispersion energy 

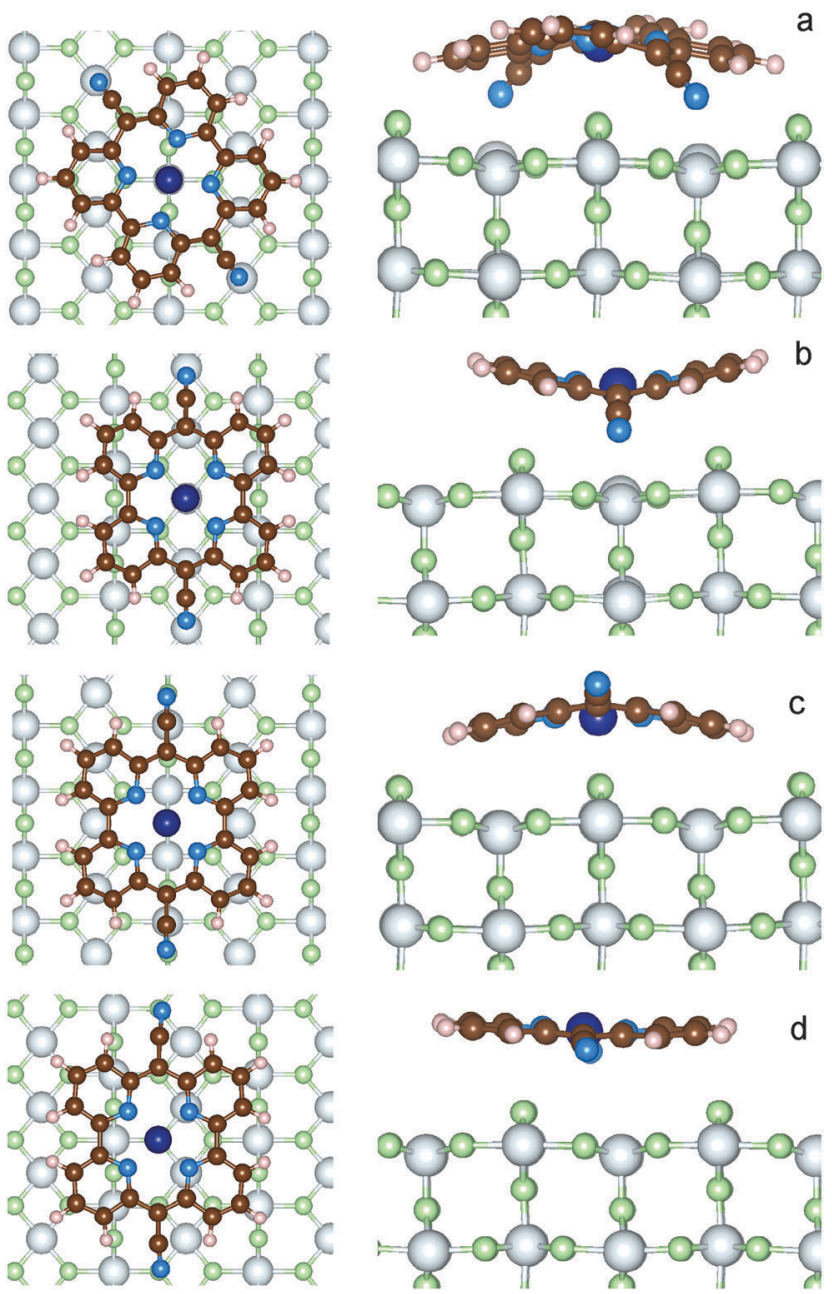

Fig. 5 Four different optimized structures of the CoPy@rutile complex, as obtained from different initial positions of the molecule. The top view (left) and one side view (right) of each of the four models are shown. Color code: see previous figures.

is extracted, so that its contribution to the adsorption energy is calculated as

$$
E_{\mathrm{ads}}^{\mathrm{disp}}=E_{\mathrm{complex}}^{\mathrm{disp}}-\left(E_{\mathrm{slab}}^{\mathrm{disp}}+E_{\mathrm{mol}}^{\mathrm{disp}}\right)
$$

The dispersion contribution is always attractive. The distortion energy of CoPy $\left(E_{\text {dist }}\right)$ is, instead, the increase in molecular energy due to the geometrical changes induced by the interaction with the surface,

$$
E_{\mathrm{dist}}=E_{\mathrm{mold}}-E_{\mathrm{mol}}
$$

$E_{\text {mold }}$ is the energy of the molecule calculated in a vacuum, but with the same coordinates obtained from the complex optimization.

\section{Results and discussion}

The resulting four adsorption configurations of CoPy@rutile are characterized by different energies and structural parameters. In Table 5, the above defined $E_{\text {ads }}, E_{\text {ads }}^{\text {disp }}, E_{\text {dist }}$ values
Table 5 Energies and structural parameters characterizing the four distinct adsorption sites reported in Fig. 5

\begin{tabular}{lllllllll}
\hline & $\begin{array}{l}E_{\text {ads }} \\
\text { Model }\end{array}$ & $\begin{array}{l}E_{\text {ads }}^{\text {disp }} \\
{[\mathrm{eV}]}\end{array}$ & $\begin{array}{l}E_{\text {dist }} \\
{[\mathrm{eV}]}\end{array}$ & $\begin{array}{l}h_{\text {av }} \\
{[\AA]}\end{array}$ & $\begin{array}{l}\Delta h \\
{[\AA]}\end{array}$ & $\begin{array}{l}d_{\mathrm{CN}} \\
{[\AA]}\end{array}$ & $\begin{array}{l}d_{\text {min }} \\
{[\AA]}\end{array}$ & Pair \\
\hline a & -4.18 & -2.62 & 0.57 & 3.67 & 2.16 & 10.86 & 2.13 & $\mathrm{~N}_{\mathrm{CN}}-\mathrm{Ti}_{5 \mathrm{c}}$ \\
$\mathrm{b}$ & -3.56 & -2.34 & 0.40 & 3.89 & 2.34 & 11.14 & 2.22 & $\mathrm{~N}_{\mathrm{CN}}-\mathrm{Ti}_{5 \mathrm{c}}$ \\
$\mathrm{c}$ & -3.35 & -2.89 & 0.24 & 3.52 & 1.66 & 11.51 & 2.25 & $\mathrm{Co}(\mathrm{II})-\mathrm{O}_{2 \mathrm{c}}$ \\
$\mathrm{d}$ & -2.49 & -2.42 & 0.09 & 3.94 & 0.95 & 11.65 & 2.77 & $\mathrm{H}-\mathrm{O}_{2 \mathrm{c}}$
\end{tabular}

are reported together with the average height of the molecule on the $\mathrm{Ti}_{5 \mathrm{c}}$ layer $\left(h_{\mathrm{av}}\right)$, the difference in height between the lowest and the highest atoms in the molecule $(\Delta h)$, which is a measure of the deviation from planarity, the distance between the two $\mathrm{N}_{\mathrm{CN}}$ atoms $\left(d_{\mathrm{CN}}\right)$, and the minimum distance between an atom of the molecule and an atom of the surface $\left(d_{\min }\right)$.

As a general observation, the interaction to the surface is relatively weak and the major contribution is due to the dispersion term, which varies from $60 \%$ to almost $100 \%$ of the adsorption energy. The strongest adsorption is obtained for model-a, where the molecule is bent along the $d_{\mathrm{CN}}$ axes such that both the $\mathrm{N}_{\mathrm{CN}}$ atoms can sit exactly on top of $\mathrm{Ti}_{5 \mathrm{c}}$ centers, while $\mathrm{Co}(\mathrm{II})$ coordinates two $\mathrm{O}_{2 \mathrm{c}}$. The interaction is further favored by the upward displacement of the involved Ti atoms by $0.26 \AA$, thus reducing the N-Ti distance to only $2.13 \AA$. CoPy is bent along both axes, even if the curvature is more pronounced along $d_{\mathrm{CN}}$, reducing the $d_{\mathrm{CN}}$ distance by about $1 \AA$ with respect to the gas phase structure. The geometrical changes correspond to a distortion energy of $0.57 \mathrm{eV}$. Subtracting from the total adsorption energy the distortion energy, we obtain an interaction energy of $-4.75 \mathrm{eV}$, of which $-2.62 \mathrm{eV}$ are attributed to dispersion. The remaining $-2.13 \mathrm{eV}$ are mainly due to the chemical bonding between $\mathrm{N}_{\mathrm{CN}}$ and $\mathrm{Ti}\left(\approx-1 \mathrm{eV}\right.$ per $\left.\mathrm{N}_{\mathrm{CN}}\right)$, which, as it will be discussed in the following, is responsible for the rehybridization of the molecular HOMO with the surface states.

In model-b, both $\mathrm{N}_{\mathrm{CN}}$ and $\mathrm{Co}(\mathrm{II})$ are sitting on top of $\mathrm{Ti}_{5 \mathrm{c}}$ centers, and the $d_{\mathrm{CN}}$ axis is oriented along the [001] lattice direction, i.e. rotated by about 30 degrees with respect to model-a. Also in this case, the closest contact between the molecule and the surface is the $\mathrm{N}-\mathrm{Ti}$ interaction, and the involved $\mathrm{Ti}$ atoms are displaced upwards by $0.15 \AA$ A. However, in this position, some $\mathrm{O}_{2 \mathrm{c}}$ atoms happen to be in proximity of the pyridine rings, which consequently bend outwards. Overall, the distance between the molecule and the surface ( $\left.h_{\mathrm{av}}=3.89 \AA\right)$ is larger than in model-a, and, even though the molecular distortion energy is smaller, the adsorption interaction is weaker. This can be explained in terms of the repulsive electrostatic interaction between $\mathrm{Co}$ (II) and $\mathrm{Ti}$ and the reduced dispersion contribution due to the larger average distance.

Like in model-a, in model-c Co coordinates the two closest dangling $\mathrm{O}$. The $\mathrm{N}_{\mathrm{CN}}$ are on top of the surface oxygen giving rise to a repulsive interaction, which causes the upwards bending along the $d_{\mathrm{CN}}$ axis. Since only the cyano groups are displaced, the molecule is less distorted than in the two previous configurations, as also indicated by its $d_{\mathrm{CN}}$ length and the computed distortion energy. In average, CoPy is closer to the surface and, 
as a consequence, the dispersion energy term is larger. By subtracting the distortion energy and the dispersion energy from the total adsorption energy, the remaining interaction energy amounts to $0.70 \mathrm{eV}$.

Finally, in model-d, the most active molecular centers, Co(II) and $\mathrm{N}_{\mathrm{CN}}$, are too far from the under-coordinated atoms at the surface. The molecular structure is only slightly modified upon adsorption, since the only attractive interaction is attributed to the dispersion forces. This is a classical example of physisorption, as also confirmed by the almost equal values of $E_{\text {ads }}$ and $E_{\text {ads }}^{\text {disp }}$. Rather weak physisorption has been already reported for the case of a single pyridine ring adsorbed on $\mathrm{TiO}_{2} \cdot{ }^{67}$

The standing adsorption of CoPy on the rutile(110) surface has also been examined. CoPy is positioned perpendicular to the (110) surface, with one $\mathrm{N}_{\mathrm{CN}}$ on top of one $\mathrm{Ti}_{5 \mathrm{c}}$, since between these two species the binding is expected to be strongest. After the optimization of the structure, the distance between $\mathrm{N}_{\mathrm{CN}}$ and $\mathrm{Ti}_{5 \mathrm{c}}$ is $2.09 \AA$. The resulting adsorption energy $E_{\text {ads }}$ is $-1.61 \mathrm{eV}$, i.e. $2.5 \mathrm{eV}$ lower than for the molecule adsorbed flat. The obvious reasons for the weaker binding are that only one direct $\mathrm{N}_{\mathrm{CN}}-\mathrm{Ti}_{5 \mathrm{c}}$ interaction is available and the significantly smaller contribution of dispersion $(-0.65 \mathrm{eV})$, given the standing geometry.

Pratik et $a{ }^{68}{ }^{6}$ modeled several different porphyrin-derived molecules adsorbed both horizontally and vertically on anatase nanoparticles. Their calculations show that while horizontal orientation of porphyrin on anatase has $E_{\text {ads }}$ of $-2.6 \mathrm{eV}$, porphyrin-4- $\mathrm{NO}_{2}$ has $-9.30 \mathrm{eV}$, and porphyrin-4- $\mathrm{NH}_{2}$ has $-4.33 \mathrm{eV}$, vertical attachment of $1-\mathrm{NMe}_{2}-$ porphyrin-1-COOH results in $E_{\text {ads }}$ of $-2.80 \mathrm{eV}$.

In summary, the most effective interactions for the adsorption of CoPy on rutile are those between $\mathrm{N}_{\mathrm{CN}}$ and the $\mathrm{Ti}_{5 \mathrm{c}}$ surface atoms, followed by the $\mathrm{Co}(\mathrm{II})-\mathrm{O}_{2 \mathrm{c}}$ coordination. These are maximized in model-a, which turns out to be the most stable adsorption site. Therefore, the following analysis of the electronic properties of the CoPy@rutile is based on this model.

The redistribution of the electronic charge induced by the adsorption is shown in the electron density difference maps. The electron density difference is calculated by subtracting from the total density of the complex $\rho_{\text {complex }}(\mathbf{r})$ the individual electron densities of CoPy and the slab, at the same coordinates as in the complex

$$
\Delta \rho(\mathbf{r})=\rho_{\text {complex }}(\mathbf{r})-\left(\rho_{\text {slab }}(\mathbf{r})+\rho_{\text {mol }}(\mathbf{r})\right) .
$$

The two isosurfaces of $\Delta \rho(\mathbf{r})$ in Fig. 6 correspond to +0.317 e $\AA^{-3}$, i.e., charge accumulation (top left panel), and -0.317 e $\AA^{-3}$ (top right panel), i.e., charge depletion. We notice that the electrons on the pyridine rings are partially pushed away from the surface, to reduce the overlap with dangling orbitals of the $\mathrm{O}_{2 \mathrm{c}}$ atoms. On the other hand, electron accumulation is observed between $\mathrm{N}_{\mathrm{CN}}$ and the closest under-coordinated $\mathrm{Ti}_{5 \mathrm{c}}$ atoms, which confirms the presence of a chemical interaction between these species. The rearrangement leads to the polarization of the molecule along the perpendicular axis $z$. In panel c of Fig. 6 the 2D-integrated density difference is calculated as

$$
\Delta \rho_{x y}(z)=\int \mathrm{d} x \int \mathrm{d} y \Delta \rho(\mathbf{r})
$$
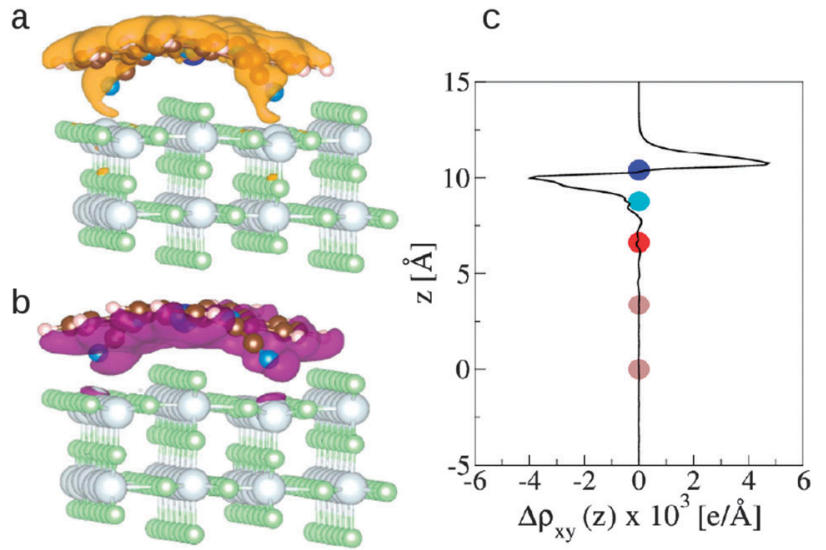

Fig. 6 Electron density difference $\Delta \rho(\mathbf{r})$ : isosurfaces taken at 0.317 e $\AA^{-3}$ (a) and -0.317 e $\AA^{-3}$ (b). (c) $2 D$ integrated density difference $\Delta \rho_{x y}(z)$ plotted along the slab's perpendicular axis. Red sphere indicates the height of the under-coordinated $\mathrm{Ti}_{5 \mathrm{c}}$ atoms, the cyan is $\mathrm{N}_{\mathrm{CN}}$, the dark blue Co(II), and the brown spheres are located at the height of two deeper layers.

is plotted along $z$. We observe that a finite dipole moment arises along the $z$ axis, which, estimated as $\mu_{z}=\int \rho_{x y}(z) \cdot z \mathrm{~d} z$, amounts to $0.5 \mathrm{D}$.

The total and projected densities of states, TDOS and PDOS, provide further information on the redistribution of the molecular orbitals upon adsorption and on the nature of the interaction. Spin polarization has been used for all calculations; however, not negligible spin density difference is observed only on Co(II) (see ESI $\dagger$ ). Therefore, we plot the distinct alpha and beta spin DOS solely for $\mathrm{Co}(\mathrm{II})$. We compare the density of states calculated for the CoPy@rutile complex with those calculated for the optimized molecule in the gas phase and for the pristine rutile surface. All PDOS plots related to the complex are aligned with respect to the corresponding VBM and normalized by the number of atoms considered in the projection.

In Fig. 7, the TDOS of CoPy@rutile (top panel) is compared to the contribution obtained from its projection on the CoPy molecule only (bottom panel). The different normalization factor makes the low intensity features of the molecular states appear with more evidence in the bottom panel. The HOMO of the complex is clearly a molecular state of CoPy. The LUMO, on the other hand, is mainly located on the rutile slab. The calculated energy gap turns out to be $1.06 \mathrm{eV}$, which is $1.22 \mathrm{eV}$ smaller than the gap of rutile(110). The lowest unoccupied states with major contributions on CoPy are about $2.5 \mathrm{eV}$ above the VBM.

The effects of CoPy adsorption on the DOS of the slab are evaluated by projecting only on the slab's atoms and comparing to the PDOS computed for the pristine slab. In Fig. 8, we distinguish between contributions from surface titanium atoms (b), and surface oxygen atoms (c). The signature of the surface $\mathrm{Ti}_{5 \mathrm{c}}$ atoms is the growth in intensity of the left shoulder of the lowest band of unoccupied states, as compared to PDOS of bulk Ti atoms (see Fig. 1). The center of this band is at about $1.5 \mathrm{eV}$. Of the $21 \mathrm{Ti}_{5 \mathrm{c}}$ atoms at the surface in our model slab, 


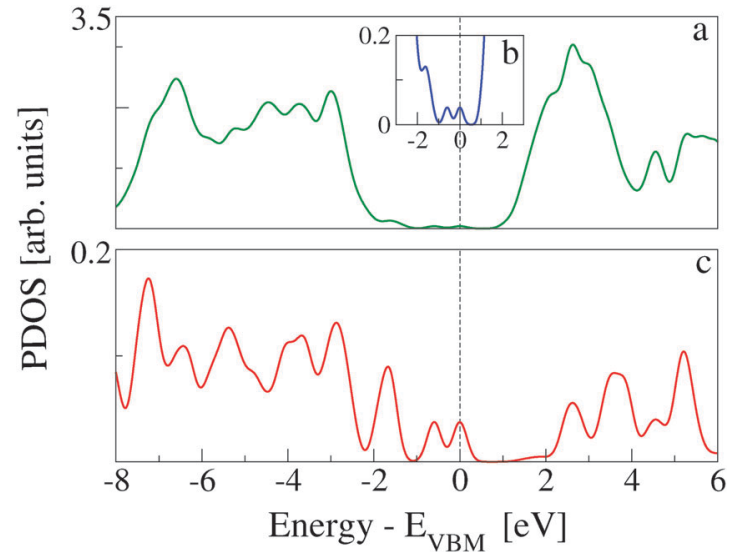

Fig. 7 (a) TDOS of CoPyarutile(110). The inset (b) shows a zoom-in of the energy gap region, which allows us to distinguish the molecular states within the rutile energy gap. (c) DOS projected on all the atoms of CoPy. Since the number of atoms is much smaller, the normalization factor is different, which allows us to better appreciate the fine structure of the molecular states.

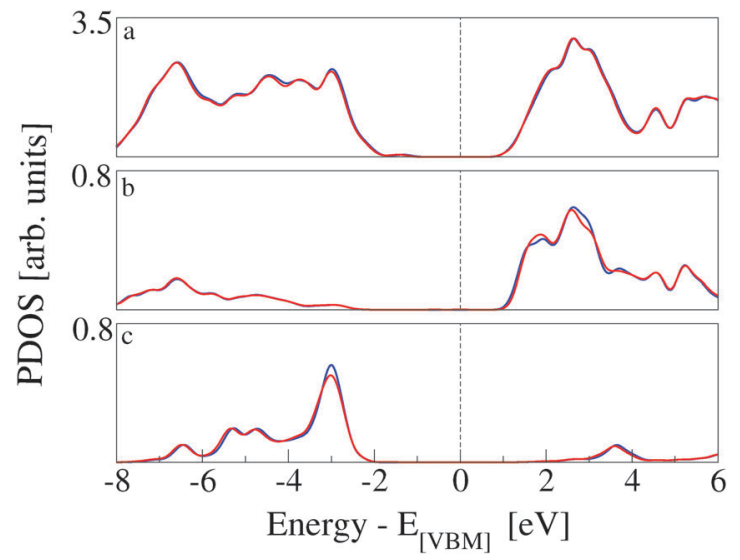

Fig. 8 PDOS related to surface Ti and O atoms of CoPy@rutile(110) (red) and of the clean rutile(110) (blue) are compared. While energy states of CoPy arutile are aligned with respect to its VBM, alignment in pristine rutile is carried out with respect to the highest occupied band of surface $O$ atoms of CoPy@rutile. (a) TDOS. (b) PDOS on surface under-coordinated Ti centers. (c) PDOS on under-coordinated $O$ centers at the surface.

only two strongly interact with the molecule via the $\mathrm{N}_{\mathrm{CN}}$ linkers and are responsible for the small difference between the red (CoPy@rutile) and the blue (clean) curves in panel b. The band of the highest occupied states in rutile is mainly constituted of O-2p orbitals. The differences between the bulk (see Fig. 1) and the surface are more evident in this case since the surface oxygen PDOS present one relatively narrow band at about $-3 \mathrm{eV}$ and lower intensity features at lower energies. This pronounced feature is formed by the highest occupied surface states. The effects of CoPy adsorption in the $\mathrm{O}_{2 \mathrm{c}}$ PDOS are ascribed to the two surface oxygens interacting with $\mathrm{Co}(\mathrm{II})$.

The electronic structure rearrangements occurring upon adsorption have their major effects on the CoPy molecular orbitals. The PDOS on CoPy adsorbed and in the gas phase are compared in Fig. 9. All the plots are aligned with the HOMO at zero eV. The PDOS of CoPy@rutile (red curves) shows a

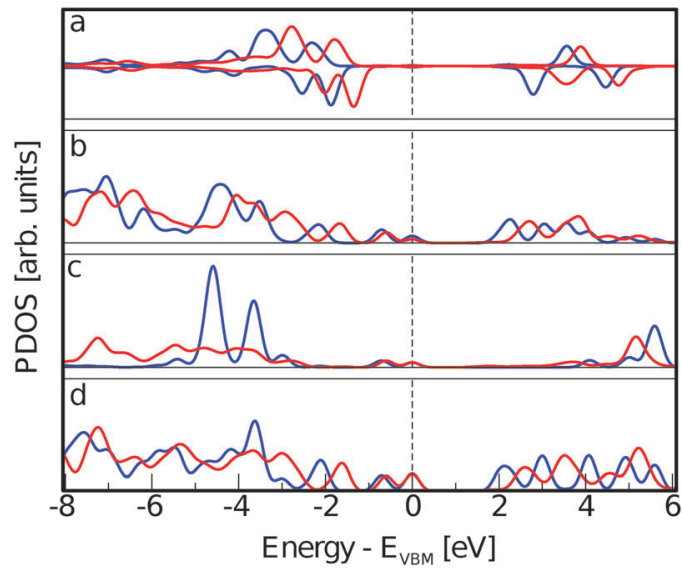

Fig. 9 PDOS on different species of CoPy adsorbed on rutile (red solid line) and optimized in the gas phase (blue solid line). (a) PDOS on Co. Positive values are for the alpha spin channel and negative values for the beta spin channel. (b) PDOS on the central $\mathrm{N}$ atoms binding to the pyridine rings, $N_{p}$. (c) PDOS on the external $N$ atoms belonging to the cyano groups, $N_{C N}$. (d) PDOS on all carbon atoms.
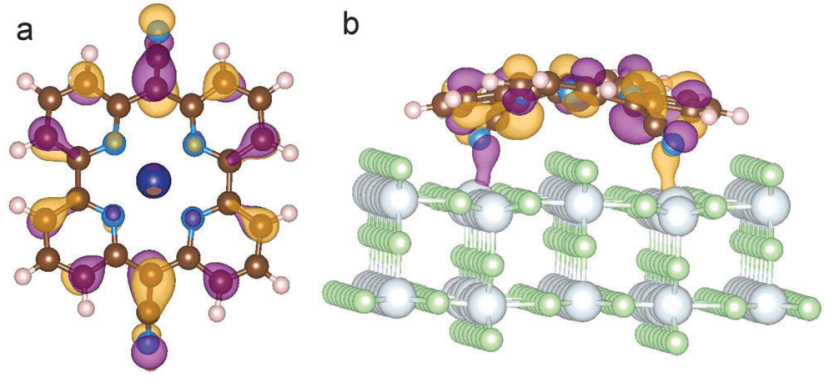

Fig. 10 Representation of the HOMO of CoPy adsorbed on rutile(110). Top (a) and side (b) view. The displayed isosurfaces are obtained at \pm 0.236 e $\AA^{-3}$.

general broadening of the bands. The occupied states on Co (a), $\mathrm{N}_{\mathrm{p}}$ (b), and C (d) present the same main features as in the gas phase. The lowest unoccupied states are shifted to higher energies by about $0.5 \mathrm{eV}$. The projected energy gap becomes $0.48 \mathrm{eV}$ larger than the HOMO-LUMO gap in the gas phase, if one considers the lowest unoccupied state with major contributions on the adsorbed molecule, which actually is at $2.5 \mathrm{eV}$ above the HOMO. The sharp bands characterizing the PDOS on the $\mathrm{N}_{\mathrm{CN}}$ in the gas phase are most strongly affected by the interaction with the surface. The two localized molecular states at $-3.7 \mathrm{eV}$ and $-5.5 \mathrm{eV}$ are replaced by a broad distribution due to the re-hybridization with the Ti states. Moreover, the interaction has a clear effect on the charge distribution corresponding to the HOMO of CoPy. As shown in Fig. 10, the orbital extends in the region between the molecule and the surface, thus strengthening the chemical bonding. This electronic rearrangement explains the bending along $d_{\mathrm{CN}}$ and the upward displacement of the Ti atoms.

Finally, we separate the contributions to the DOS ascribed to the two $\mathrm{Ti}_{5 \mathrm{c}}$ and the two $\mathrm{O}_{2 \mathrm{c}}$ atoms more directly involved in the interaction. The resulting PDOS are plotted in Fig. 11 and 


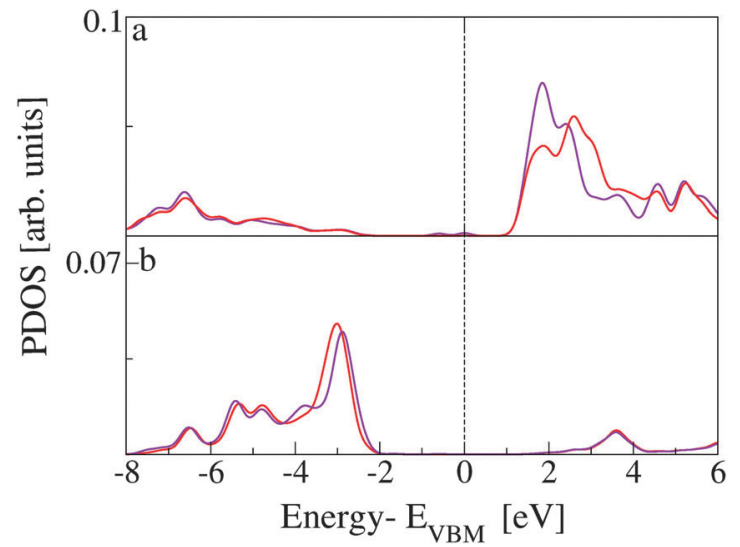

Fig. 11 (a) PDOS on $\mathrm{Ti}_{5 \mathrm{c}}$ atoms. The red line is the same PDOS shown in panel $b$ of Fig. 8, related to the complex. The purple line is related to the only two surface Ti atoms interacting directly with $\mathrm{N}_{\mathrm{CN}}$. (b) PDOS on $\mathrm{O}_{2 c}$ atoms. Red line is the same PDOS presented in panel c of Fig. 8. The purple line is obtained by the projection on the two oxygen atoms coordinating the Co(II) in CoPyarutile(110).

compared with the PDOS obtained from all the $\mathrm{Ti}_{5 \mathrm{c}}$ and $\mathrm{O}_{2 \mathrm{c}}$ present at the slab surface. While no significant difference is observed among the occupied states, a clear effect of the interaction is present for the lowest unoccupied states localized at $\mathrm{Ti}_{5 \mathrm{c}}$. In particular, we observe the increase in intensity of the first peak and the consequent depletion at higher energies. This result implies the presence of available empty states on the $\mathrm{Ti}$ atoms that are also involved in the extension of the molecular HOMO state. On the other hand, the occupied states localized on the surface $\mathrm{O}$ atoms are more than $2 \mathrm{eV}$ lower in energy than the CoPy HOMO and are not significantly perturbed by the adsorbate.

\section{Conclusions}

We have investigated the adsorption of a porphyrin derived heterocyclic molecule, CoPy, on the rutile(110) surface. This new type of heterocyclic molecule has been experimentally proved to be an active catalyst for hydrogen evolution reactions. However, there are no extensive studies on its properties and, to our knowledge, this is the first time that the adsorption of one of these has been considered. We observe electronic structure rearrangements occurring upon adsorption, mainly due to the interaction of the cyano groups of CoPy with the fivecoordinated Ti atoms of rutile(110). The projected densities of states on the different involved species reveal that the molecular orbitals on the $\mathrm{N}_{\mathrm{CN}}$ atoms undergo a rehybridization, the HOMO is located at the molecule, and the lowest unoccupied states of CoPy are shifted to higher energies. Effects of the electronic redistribution are also recognized from the $\mathrm{Ti}_{5 \mathrm{c}}$ PDOS and the polarization of the electronic density across the molecular plane. The first band of unoccupied states is mainly on rutile and extends from 1.1 to $2.0 \mathrm{eV}$, with a maximum at $1.5 \mathrm{eV}$. The lowest unoccupied states localized on CoPy are instead beyond $2.5 \mathrm{eV}$, i.e., at higher energy with respect to the
LUMO of the gas phase molecule. Hence, the adsorption produces a significant reduction of the energy gap with respect to both pristine rutile $\mathrm{TiO}_{2}$ and the isolated molecule, which should facilitate absorption of light in the visible range. This opens up the possibility of achieving electron injection from CoPy to rutile, with a consequent efficient electron-hole separation.

On the other hand, but for a rigid shift with respect to the HOMO, we do not observe strong modifications of the electronic structure at the $\mathrm{Co}(\mathrm{II})$ center. From these results we expect that the catalytic properties of CoPy are preserved upon adsorption. We plan to extend the study on this system also considering the co-adsorption of water and hydrogen and possible reaction mechanisms leading to the production of molecular hydrogen. We expect that by functionalizing pyrphyrin with different linkers and replacing Co with other transition metals, a further tuning of the coupling with the oxide is possible and improved water splitting performance can be achieved.

\section{Acknowledgements}

We gratefully acknowledge the financial support from the University of Zurich, the University Research Priority Program "Solar Light to Chemical Energy Conversion" (LightChEC), National Centres of Competence in Research-Materials Revolution: Computational Design and Discovery of Novel Materials (NCCRMARVEL), and computing resources from the Swiss National Supercomputer Centre (CSCS) under the project ID s425.

\section{References}

1 W. T. Eckenhoff and R. Eisenberg, Dalton Trans., 2012, 41, 13004.

2 N. Armaroli and V. Balzani, Angew. Chem., Int. Ed., 2007, 46, 52 .

3 M. Carmo, D. L. Fritz, J. Mergel and D. Stolten, Int. J. Hydrogen Energy, 2013, 38, 4901.

4 T. L. LeValley, A. R. Richard and M. Fan, Int. J. Hydrogen Energy, 2014, 39, 16983.

5 E. Seker, Int. J. Hydrogen Energy, 2008, 33, 2044.

6 A. T. Ashcroft, A. K. Cheetham, J. S. Foord, M. L. H. Green, C. P. Grey, A. J. Murrell and P. D. F. Vernon, Nature, 1990, 344, 319.

7 S. G. Avcioglu, E. Ozgur, I. Eroglu, M. Yucel and U. Gunduz, Int. J. Hydrogen Energy, 2011, 36, 11360.

8 M. Grätzel, Nature, 2001, 414, 338.

9 M. Wang, Y. Na, M. Gorlov and L. Sun, Dalton Trans., 2009, 6458.

10 A. J. Esswein and D. G. Nocera, Chem. Rev., 2007, 107, 4022.

11 S. Losse, J. G. Vos and S. Rau, Coord. Chem. Rev., 2010, 254, 2492.

12 X. Hu, B. S. Brunschwig and J. C. Peters, J. Am. Chem. Soc., 2007, 129, 8988.

13 M. Guttentag, A. Rodenberg, R. Kopelent, B. Probst, C. Buchwalder, M. Brandstätter, P. Hamm and R. Alberto, Eur. J. Inorg. Chem., 2012, 59. 
14 H. Imahori and S. Fukuzumi, Adv. Funct. Mater., 2004, 14, 525.

15 L. Si and H. He, J. Phys. Chem. A, 2014, 118, 3410.

16 B. H. Solis, A. G. Maher, T. Honda, D. C. Powers, D. G. Nocera and S. Hammes-Schiffer, ACS Catal., 2014, 4, 4516.

17 S. Soman, Comments Inorg. Chem., 2015, 35, 82.

18 Z. Han and R. Eisenberg, Acc. Chem. Res., 2014, 47, 2537.

19 S. Berardi, S. Drouet, L. Francas, C. Gimbert-Surinach, M. Guttentag, C. Richmond, T. Stoll and A. Llobet, Chem. Soc. Rev., 2014, 43, 7501.

20 A. Rodenberg, M. Orazietti, B. Probst, C. Bachmann, R. Alberto, K. K. Baldridge and P. Hamm, Inorg. Chem., 2015, 54, 646.

21 C. Kutal, J. Chem. Educ., 1983, 60, 882.

22 C. G. Garcia, J. F. de Lima and N. Y. M. Iha, Coord. Chem. Rev., 2000, 196, 219.

23 R. L. Milot, G. F. Moore, R. H. Crabtree, G. W. Brudvig and C. A. Schmuttenmaer, J. Phys. Chem. C, 2013, 117, 21662.

24 H. Imahori, S. Kang, H. Hayashi, M. Haruta, H. Kurata, S. Isoda, S. E. Canton, Y. Infahsaeng, A. Kathiravan, T. Pascher, P. Chábera, A. P. Yartsev and V. Sundström, J. Phys. Chem. A, 2011, 115, 3679.

25 L.-L. Li and E. W.-G. Diau, Chem. Soc. Rev., 2013, 42, 291.

26 F. Lakadamyali and E. Reisner, Chem. Commun., 2011, 47, 1695.

27 M. Yin, S. Ma, C. Wu and Y. Fan, RSC Adv., 2015, 5, 1852.

28 F. Lakadamyali, A. Reynal, M. Kato, J. R. Durrant and E. Reisner, Chem. - Eur. J., 2012, 18, 15464.

29 D. Achey, S. Ardo and G. J. Meyer, Inorg. Chem., 2012, 51, 9865.

30 S. Ogawa, R. Narushima and Y. Arai, J. Am. Chem. Soc., 1984, 106, 5760.

31 S. Ogawa, T. Uchida, T. Uchiya, T. Hirano, M. Saburi and Y. Uchidac, J. Chem. Soc., Perkin Trans. 1, 1990, 1649.

32 C. Pierre, J.-M. Vincent, J.-B. Verlhac, C. Courseille, A. Dautant and C. Mathoniere, New J. Chem., 2001, 25, 522.

33 Z. Zhu, K. Takano, A. Furuhama, S. Ogawa and S. Tsuchiya, Bull. Chem. Soc. Jpn., 2007, 80, 686.

34 R. Ibrahim, S. Tsuchiya and S. Ogawa, J. Am. Chem. Soc., 2000, 122, 12174.

35 L. Tim, H. Sandamali, T. Junguang, K. Alan, S. Eli and B. Matthias, Sci. Rep., 2014, 4, 4043.

36 J. Chen, Y.-F. Li, P. Sit and A. Selloni, J. Am. Chem. Soc., 2013, 135, 18774.

37 A. G. Thomas and K. L. Syres, Chem. Soc. Rev., 2012, 41, 4207.

38 J. VandeVondele, M. Krack, F. Mohamed, M. Parrinello, T. Chassaing and J. Hutter, Comput. Phys. Commun., 2005, 167, 103.

39 J. VandeVondele and J. Hutter, J. Chem. Phys., 2007, 127, 114105.

40 S. Goedecker, M. Teter and J. Hutter, Phys. Rev. B: Condens. Matter Mater. Phys., 1996, 54, 1703.

41 J. P. Perdew, K. Burke and M. Ernzerhof, Phys. Rev. Lett., 1996, 77, 3865.
42 S. Grimme, J. Antony, S. Ehrlich and H. Krieg, J. Chem. Phys., 2010, 132, 154104.

43 T. L. Bahers, M. Rérat and P. Sautet, J. Phys. Chem. C, 2014, 118, 5997.

44 A. J. Cohen, P. Mori-Sánchez and W. Yang, Science, 2008, 321, 792.

45 J. Muscat, V. Swamy and N. M. Harrison, Phys. Rev. B: Condens. Matter Mater. Phys., 2002, 65, 224112.

46 F. Labat, P. Baranek and C. Adamo, J. Chem. Theory Comput., 2008, 4, 341.

47 H. Xiao, J. Tahir-Kheli and W. A. Goddard, J. Phys. Chem. Lett. , 2011, 2, 212.

48 J. Heyd, G. E. Scuseria and M. Ernzerhof, J. Chem. Phys., 2003, 118, 8207.

49 A. V. Krukau, O. A. Vydrov, A. F. Izmaylov and G. E. Scuseria, J. Chem. Phys., 2006, 125, 224106.

50 M. Guidon, J. Hutter and J. VandeVondele, J. Chem. Theory Comput., 2010, 6, 2348.

51 M. Guidon, J. Hutter and J. VandeVondele, J. Chem. Theory Comput., 2009, 5, 3010.

52 M. Guidon, F. Schiffmann, J. Hutter and J. VandeVondele, J. Chem. Phys., 2008, 128, 214104.

53 C. J. Howard, T. M. Sabine and F. Dickson, Acta Crystallogr., Sect. B: Struct. Sci., 1991, 47, 462.

54 J. K. Burdett, T. Hughbanks, G. J. Miller, J. W. Richardson and J. V. Smith, J. Am. Chem. Soc., 1987, 109, 3639.

55 H. Perron, J. Vandenborre, C. Domain, R. Drot, J. Roques, E. Simoni, J. J. Ehrhardt and H. Catalette, Surf. Sci., 2007, $601,518$.

56 Z. Y. Zhao, J. Phys. Chem. C, 2014, 118, 4287.

57 H. Perron, C. Domain, J. Roques, R. Drot, E. Simoni and H. Catalette, Theor. Chem. Acc., 2007, 117, 565.

58 L. Kavan, M. Grätzel, S. E. Gilbert, C. Klemenz and H. J. Scheel, J. Am. Chem. Soc., 1996, 118, 6716.

59 R. Evarestov and Y. Zhukovskii, Surf. Sci., 2013, 608, 226.

60 U. Diebold, Surf. Sci. Rep., 2003, 48, 53.

61 C. Lun Pang, R. Lindsay and G. Thornton, Chem. Soc. Rev., 2008, 37, 2328.

62 S.-C. Li and U. Diebold, J. Am. Chem. Soc., 2010, 132, 64.

63 T. R. Esch, I. Gadaczek and T. Bredow, Appl. Surf. Sci., 2014, 288, 275.

64 S. Fujiyoshi, T.-A. Ishibashi and H. Onishi, J. Phys. Chem. B, 2005, 109, 8557.

65 A. T. Paxton and L. Thiên-Nga, Phys. Rev. B: Condens. Matter Mater. Phys., 1998, 57, 1579.

66 We also optimized the structure of the second isomer of metal-free pyrphyrin finding that when the two protons reside on the same side the molecule is by $0.23 \mathrm{eV}$ less stable.

67 S. Suzuki, Y. Yamaguchi, H. Onishi, T. Sasaki, K. Fukui and Y. Iwasawa, J. Chem. Soc., Faraday Trans., 1998, 94, 161.

68 S. M. Pratik and A. Datta, Phys. Chem. Chem. Phys., 2013, 15, 18471. 